\section{Contexto sócio-econômico e percepção da saúde bucal em uma população de adultos no Rio de Janeiro, Brasil: uma análise multinível}

\author{
Socioeconomic context and perceived oral \\ health in an adult population in Rio de Janeiro, \\ Brazil: a multilevel analysis
}

\footnotetext{
${ }^{1}$ Instituto de Medicina Social, Universidade do Estado do Rio de Janeiro, Rio de Janeiro, Brasil. 2 Escola Nacional de Saúde Pública Sergio Arouca, Fundação Oswaldo Cruz, Rio de Janeiro, Brasil. 3 Núcleo de Estudos de Saúde Coletiva, Universidade Federal do Rio de Janeiro, Rio de Janeiro, Brasil.

Correspondência P. Nadanovsky Departamento de Epidemiologia, Instituto de Medicina Social, Universidade do Estado do Rio de Janeiro.

Rua São Francisco Xavier 524 70 andar. Rio de Janeiro, $R J$ 20559-900, Brasil. nadanovsky@ims.uerj.br
}

\begin{abstract}
One of the main limitations of the ecological design is aggregation bias. The problem can be addressed by designs that use small contextual hierarchical units with internal homogeneity. This study aimed to compare different geographic data structures in order to explore contextual effects of socioeconomic variables on the odds of perceived poor oral health. The analyses were based on a mixed design that included data from civil servants participating in the "Pró-Saúde" censuses I and II and residing in the city of Rio de Janeiro, Brazil, in 1999 ( $n=2,426)$; contextual data were obtained from the National Demographic Census conducted in 1991. We used the variance partition coefficient to compare different geographic hierarchical structures. In this study, the smallest geographic partition (census tract) was the best unit for contextual analysis for studying variability in perceived poor oral health. Individual socioeconomic attributes explained more of the variance in perceived oral health than did the socioeconomic contexts of residential areas.
\end{abstract}

Oral Health; Socioeconomic Analysis; Adult

\author{
Wagner de Souza Tassinari 1,2 \\ Antônio Ponce de León 1 \\ Guilherme Loureiro Werneck 1,3 \\ Eduardo Faerstein 1 \\ Claudia S. Lopes 1 \\ Dora Chor 2 \\ Paulo Nadanovsky 1
}

\section{Introdução}

Quando o objetivo central de um estudo epidemiológico é investigar efeitos do meio social, econômico ou ambiental na saúde de uma população, estudos ecológicos são considerados os delineamentos mais apropriados 1. Porém, muito se debate a respeito da fragilidade dos desenhos ecológicos quando se quer estimar o efeito contextual de uma exposição na saúde de indivíduos como, por exemplo, o efeito da poluição ambiental na ocorrência de doenças respiratórias. Tal exposição é comum aos indivíduos que fazem parte de uma mesma base territorial (geográfica) ou institucional. Delineamentos ecológicos são mais susceptíveis a vieses como, por exemplo, o viés de agregação, também denominado de falácia ecológica, decorrente da suposição de que atributos de uma determinada área aplicam-se de forma homogênea a todos os indivíduos dessa mesma área 2. Problemas desse tipo podem ser minimizados por meio da escolha de níveis de hierarquia, compostos por unidades (grupos, agregados ou clusters) de tamanho adequado de forma a produzir a homogeneidade interna desejada ${ }^{3}$. Apesar de tais inconveniências, deve-se ter em mente que atributos no nível individual podem não ser suficientes para explicar o processo de adoecimento, uma vez que dentro do contexto comunitário existem fatores culturais e geofísicos (por exemplo, relevo e condições climáticas), dentre ou- 
tros, que podem afetar, direta ou indiretamente, o indivíduo.

Indivíduos são reunidos em grupos, cujas propriedades não são apenas somatórios das mensurações de cada um dos seus respectivos membros. Diante desse cenário, é importante estimar os efeitos contextuais que podem afetar os indivíduos. Para que isso seja viável, deve-se dispor tanto de informações individuais quanto dos respectivos grupos, formando um banco de dados com estrutura hierárquica. Para analisar esses dados, é necessário que se leve em conta a estrutura de dependência entre dados individuais e seus respectivos grupos de origem 1, o que é possível por meio de modelos hierárquicos 4 .

Uma das vantagens da aplicação de modelos hierárquicos é a garantia de um melhor tratamento entre grupos mais similares que outros, pois sua estrutura é bastante flexível quando existe heterogeneidade entre os grupos. Todas essas justificativas devem-se à capacidade desse tipo de modelo de decompor a estrutura de variância das observações, em diferentes níveis de hierarquia 5. Em estatística espacial, o fenômeno da homogeneidade das unidades hierárquicas geográficas tem sido explorado há bastante tempo 6 . Nessa área do conhecimento, o fenômeno que retrata esta inter-relação entre a área de estudo e o resultado mensurado é chamado de MAUP ( $\mathrm{Mo}$ difiable Area Unit Problem - problema da unidade de área modificável). Nesse caso, mudanças na escala e nas dimensões da unidade de análise podem levar a conclusões diferentes. Situações desse tipo são ilustradas no presente estudo.

Variáveis sócio-econômicas, demográficas e comportamentais no nível individual influenciam a saúde bucal, entretanto, pouco foi explorado sobre o efeito de variáveis contextuais $6,7,8,9,10,11,12,13$. O objetivo deste trabalho foi comparar diferentes estruturas geográficas de dados (regiões administrativas, bairros e setores censitários), com a finalidade de investigar efeitos de variáveis sócio-econômicas contextuais na chance de cada indivíduo perceber sua saúde bucal como ruim, controlando por fatores sócioeconômicos individuais.

\section{Método}

\section{Dados e população de estudo}

Dados individuais foram obtidos dos censos saúde (Estudo Pró-Saúde), realizados em 1999 e 2001 14. A população deste estudo foi composta por 2.426 indivíduos que participaram de ambos os censos e responderam à pergunta sobre saúde bucal percebida. Eles residiam em 1.640 setores censitários, compreendidos em 139 bairros distribuídos em 26 regiões administrativas do Município do Rio de Janeiro, Brasil, segundo a definição político-administrativa do Censo Demográfico de 199115.

O Censo Demográfico da Unidade Federativa do Rio de Janeiro do ano de 199115 foi utilizado para construir indicadores sócio-econômicos contextuais das áreas de residência dos indivíduos que compõem a população de estudo.

Para localizar a área de residência de cada indivíduo foi utilizado o programa de georreferenciamento de endereços SISLOC, desenvolvido pelo grupo do Laboratório de Georreferenciamento, Departamento de Informações em Saúde, Centro de Informações Científico Tecnológicas, Fundação Oswaldo Cruz, no Rio de Janeiro.

\section{Análise}

A análise estatística foi baseada em um delineamento misto abrangendo o estudo Pró-Saúde (dados sobre os indivíduos - nível individual) e o Censo Demográfico da Unidade Federativa do Rio de Janeiro do ano de 199115 (dados sobre a área de residência dos indivíduos - nível contextual). Os dados foram analisados com modelos logísticos hierárquicos e não-hierárquicos, nos quais os indivíduos foram as unidades de primeiro nível e as áreas de residência formavam as unidades do segundo e terceiro níveis. A variável desfecho foi Saúde Bucal Percebida (SBP).

Para comparar as estruturas de agregação geográfica foi estimado o Coeficiente de Partição de Variância (CPV). Essa estatística mensura a quantidade de variação de desfechos categóricos entre diferentes estruturas contextuais de dados, obtida a partir de um modelo logístico hierárquico ajustado com somente o intercepto (modelo vazio) 16. A variável SBP foi dicotomizada da seguinte forma: "SBP boa" correspondeu aos indivíduos que relataram saúde bucal "boa” ou "muito boa"; e "SBP ruim" aos que relataram saúde bucal "regular", "ruim" ou "muito ruim" 17.

As variáveis nos níveis individuais e contextuais foram agrupadas em cinco blocos: variáveis individuais demográficas (sexo e idade), individuais sócio-econômicas (renda e escolaridade), individuais de hábitos relacionados à saúde (visita ao dentista e tabagismo), variáveis contextuais no nível do setor censitário (abastecimento de água, proporção de chefes de família com baixo nível de escolaridade e proporção de chefes de família com baixa renda) e no nível bairro (desigualdade na renda, condições de vida e renda per capita).

O indicador de desigualdade na renda utilizado foi índice de Theil. Seu valor é compreendido 
entre 0 e 1 e quanto maior o seu valor, maior é a desigualdade/concentração da renda 18,19 . O Indicador de Condições de Vida (ICV) é construído utilizando-se uma metodologia idêntica à utilizada na construção do Índice de Desenvolvimento Humano (IDH), incorporando um conjunto maior de indicadores de desempenho sócio-econômico, de modo a captar, de forma mais abrangente, o estágio do desenvolvimento social de uma comunidade. Basicamente, isso é feito pela ampliação do leque de indicadores que compõem as dimensões renda, educação e longevidade, e pela introdução de duas dimensões adicionais destinadas a retratar a situação da infância e da habitação 20 .

As variáveis nos níveis contextuais foram categorizadas segundo tercis, com exceção do percentual de domicílios com abastecimento de água, que foi categorizada de forma binária, tendo como ponto de corte o valor da mediana.

Para descrever a prevalência do desfecho dentro de cada característica individual e contextual, foram calculadas as proporções daqueles que relataram "SBP ruim", e também as odds ratio (OR brutos) e seus respectivos intervalos de $95 \%$ de confiança; testes de qui-quadrado de tendência foram utilizados para aferir o grau de associação entre o desfecho e as características dos indivíduos nos seus respectivos contextos de residências.

Com o propósito de comparar os níveis de agregação geográfica em relação à variabilidade de "SBP ruim", foram ajustados modelos vazios de regressão logística com dois níveis hierárquicos para cada estrutura de agregação geográfica. Com o objetivo de obter estimativas menos viesadas e mais precisas, foram ajustados modelos utilizando o algoritmo RIGLS (Restricted Iterative Generalised Least Squares), adotando-se o método de estimação PQL (Penalized Quasilikelihood) de segunda ordem 21.

Além dos modelos vazios, foram ajustados 12 modelos hierárquicos e não-hierárquicos, que vão desde o modelo logístico clássico até o hierárquico, incluindo as variáveis individuais e contextuais. Indexando com a letra $j$ as unidades do segundo nível da hierarquia de dados, i.e., as pequenas áreas, e com a letra $i$ as unidades do primeiro nível, i.e., os indivíduos nas suas respectivas áreas de residência, os modelos ajustados neste artigo podem ser resumidos com a seguinte formulação estatística:

$$
\log \left(\pi_{\mathrm{ij}} /\left(1-\pi_{\mathrm{ij}}\right)\right)=\beta_{0 \mathrm{j}}+\Sigma_{\mathrm{k}} \beta_{\mathrm{k}} \mathrm{X}_{\mathrm{kij} .}
$$

$\mathrm{Na}$ terminologia de modelos lineares generalizados, $\pi_{\mathrm{ij}}$ é o valor esperado da resposta relativa ao $i$-ésimo indivíduo da $j$-ésima pequena área; o termo do lado direito da equação - no qual o intercepto fixo (ou aleatório), as covariá- veis individuais $\left\{X_{k}\right\}$ e seus efeitos fixos $\left\{\beta_{k}\right\}$ estão representados - é o preditor linear; e a função logito do lado esquerdo da equação é a função de ligação entre o valor esperado da resposta e o preditor linear.

Para completar o modelo, supõe-se que a resposta binária, representada por $\mathrm{Y}_{\mathrm{ij}}$, segue uma distribuição condicional de Bernoulli com parâmetro $\pi_{\mathrm{ij}}$. Uma parte do condicionamento da distribuição depende da especificação do modelo no nível do indivíduo, enquanto a outra depende da especificação no nível contextual. Aqui, o modelo contextual é especificado como:

$$
\beta_{0 \mathrm{j}}=\gamma_{00}+\Sigma_{\mathrm{l}} \gamma_{\mathrm{l}} \mathrm{Z}_{\mathrm{lj}}+\mathrm{u}_{0 \mathrm{j}} \text {. }
$$

Nessa equação, $\gamma_{00}$ é o intercepto global (fixo), as covariáveis $\left\{Z_{1}\right\}$ representam os indicadores sócio-econômicos das áreas, os parâmetros $\left\{\gamma_{1}\right\}$ seus respectivos efeitos fixos, e assume-se que $u_{0 j}$ é o efeito aleatório associado às pequenas áreas. Assume-se também que a distribuição do efeito aleatório é normal com média zero e variância desconhecida $\sigma^{2}{ }_{\mathrm{u} 0}$. Note-se que a segunda equação faz parte do preditor linear do modelo, mas corresponde à parcela de efeitos contextuais. Essa formulação do modelo multinível separa os efeitos contextuais dos efeitos de covariáveis do nível individual.

Nos modelos clássicos, os efeitos aleatórios não são considerados, ou seja $u_{0 j}=0$. Apesar de terem sido utilizados durante muito tempo, modelos com esse nível de simplificação não se justificam mais exceto no caso da variância $\sigma^{2} \mathrm{u} 0$ ser próxima de zero.

Para o ajuste dos modelos foi adotada uma estratégia que consistiu, primeiramente, na inclusão de grupamentos de variáveis no nível individual isoladamente, e depois, no ajuste dos modelos com todas as variáveis no nível individual. Tais ajustes foram feitos em modelos nãohierárquicos e hierárquicos levando em conta três níveis de hierarquia (bairros, setores censitários e indivíduos).

\section{Resultados}

A prevalência de "SBP ruim" foi de $36 \%$. As categorias das variáveis individuais que apresentaram maior prevalência de "SBP ruim" foram as seguintes: sexo masculino; faixa etária de 50 até 59 anos; salário de até $\mathrm{R} \$ 500,00$; primeiro grau incompleto; visita ao dentista somente quando tem problemas; fumantes alguma vez na vida (Tabela 1). Em relação às variáveis contextuais, as maiores prevalências de "SBP ruim" foram encontradas em residentes de setores censitários que tinham menos de 99\% (mediana) de domicílios providos com água encanada, entre $22,2 \%$ e 
Tabela 1

Freqüência das características dos indivíduos, percentual de "SBP ruim" dentro de cada categoria, odss ratio (OR) brutos, e seus respectivos intervalos de confiança, e testes qui-quadrado de tendência com 95\% de confiabilidade.

\begin{tabular}{|c|c|c|c|c|c|c|}
\hline \multirow[t]{2}{*}{ Grupos } & \multirow[t]{2}{*}{ Variáveis individuais } & \multirow{2}{*}{$\begin{array}{c}N= \\
2.426\end{array}$} & \multirow{2}{*}{$\begin{array}{c}\text { "SBP ruim" } \\
(\%)\end{array}$} & \multicolumn{2}{|c|}{ OR } & \multirow{2}{*}{$\begin{array}{c}\chi^{2} \\
\text { (p valor) }\end{array}$} \\
\hline & & & & Bruto & IC95\% & \\
\hline \multirow[t]{9}{*}{ Demográficas } & Sexo & & & & & \\
\hline & Homem & 1.003 & 37,6 & 1,13 & $0,95-1,34$ & NA \\
\hline & Mulher (Referência) & 1.423 & 34,8 & 1,00 & & \\
\hline & Idade (anos) & & & & & \\
\hline & Até 29 (Referência) & 300 & 22,3 & 1,00 & & $<0,001$ \\
\hline & $30-39$ & 934 & 33,2 & 1,73 * & $1,27-2,34$ & \\
\hline & $40-49$ & 889 & 39,3 & 2,25 * & $1,66-3,04$ & \\
\hline & $50-59$ & 256 & 48,4 & 3,27 * & $2,27-4,71$ & \\
\hline & Mais de 60 & 47 & 46,8 & 3,06 * & $1,62-5,77$ & \\
\hline \multirow[t]{16}{*}{ Sócio-econômicas } & Renda & & & & & \\
\hline & Até $\mathrm{R} \$ 500,00$ & 92 & 62,0 & 6,34 * & $4,15-11,60$ & $<0,001$ \\
\hline & $\mathrm{R} \$ 501,00-\mathrm{R} \$ 1.000,00$ & 266 & 48,9 & 4,07 * & $2,78-5,96$ & \\
\hline & $R \$ 1.001,00-R \$ 1.500,00$ & 505 & 44,4 & 3,39 * & $2,41-4,79$ & \\
\hline & $\mathrm{R} \$ 1.501,00-\mathrm{R} \$ 2.000,00$ & 433 & 36,0 & 2,40 * & $1,68-3,42$ & \\
\hline & $\mathrm{R} \$ 2.001,00-\mathrm{R} \$ 3.000,00$ & 588 & 32,0 & 2,00 * & $1,42-2,82$ & \\
\hline & $\mathrm{R} \$ 3.001,00-\mathrm{R} \$ 4.000,00$ & 221 & 22,2 & 1,21 & $0,79-1,87$ & \\
\hline & Mais de $\mathrm{R} \$ 4.000,00$ (Referência) & 284 & 19,0 & 1,00 & & \\
\hline & Não responderam & 37 & & & & \\
\hline & Escolaridade & & & & & \\
\hline & 1o grau incompleto & 131 & 57,2 & 5,26 * & $3,39-8,21$ & $<0,001$ \\
\hline & 1ㅇ grau completo +2 o grau incompleto & 355 & 57,3 & 5,27 * & $3,71-7,44$ & \\
\hline & 2o grau completo +3 o grau incompleto & 894 & 38,9 & 2,51 * & $1,84-3,41$ & \\
\hline & 3o grau completo & 715 & 24,5 & 1,28 & $0,92-1,77$ & \\
\hline & Pós-graduação (Referência) & 311 & 20,2 & 1,00 & & \\
\hline & Não responderam & 20 & & & & \\
\hline \multirow[t]{11}{*}{ Hábitos } & Visita ao dentista & & & & & \\
\hline & Nunca vai ao dentista & 17 & 41,2 & 2,25 & $0,85-5,98$ & $<0,001$ \\
\hline & Só quando tem problemas & 783 & 53,1 & 3,65 * & $2,99-4,45$ & \\
\hline & Menos freqüentemente que 2 em 2 anos & 205 & 44,5 & 2,57 * & $1,88-3,50$ & \\
\hline & De 2 em 2 anos & 332 & 29,2 & 1,33 * & $1,01-1,75$ & \\
\hline & Pelo menos 1 vez ao ano (Referência) & 1.063 & 23,7 & 1,00 & & \\
\hline & Não responderam & 26 & & & & \\
\hline & Tabagismo & & & & & \\
\hline & Sim & 940 & 43,2 & 1,74 * & $1,46-2,06$ & NA \\
\hline & Não (Referência) & 1.376 & 30,4 & 1,00 & & \\
\hline & Não responderam & 110 & & & & \\
\hline
\end{tabular}

Fonte: Faerstein et al. 14 .

$\mathrm{NA}=$ não se aplica.

* Significativo ( $p$ valor $<0,05$ ).

$41,7 \%$ (2o tercil) de chefes de família com até sete anos de estudo e mais de 47,5\% (3o tercil) dos chefes de família que recebiam até três salários mínimos; e em residentes de bairros com o índice de Theil menor que 0,387 (lo tercil), com o ICV menor que 0,873 (1o tercil) e com a renda per capita menor que 1,69 salário mínimo (1o tercil) (Tabela 2).

Não houve associação significativa da SBP com sexo, porém a chance de "SBP ruim" aumentou diretamente com a idade e inversamente com a renda (Tabela 1). Já com relação às variá- 
Freqüência das características do local de residência dos indivíduos, percentual de "SBP ruim" dentro de cada categoria, odds ratio (OR) brutos, e seus respectivos intervalos de confiança, e testes de qui-quadrado de tendência com 95\% de confiabilidade.

\begin{tabular}{|c|c|c|c|c|c|c|}
\hline \multirow[t]{2}{*}{ Grupos } & \multirow[t]{2}{*}{ Variáveis contextuais } & \multirow{2}{*}{$\begin{array}{c}N= \\
2.426\end{array}$} & \multirow{2}{*}{$\begin{array}{c}\text { "SBP ruim" } \\
(\%)\end{array}$} & \multicolumn{2}{|c|}{ OR } & \multirow{2}{*}{$\begin{array}{c}\chi^{2} \\
\text { (p valor) }\end{array}$} \\
\hline & & & & Bruto & IC95\% & \\
\hline Variáveis no & Domicílios com abastecimento de água & & & & & \\
\hline \multirow[t]{14}{*}{ nível do setor } & $<99 \%$ & 1.141 & 35,0 & 0,93 & $0,79-1,10$ & NA \\
\hline & Z 99\% (Referência) & 1.285 & 36,8 & 1,00 & & \\
\hline & Chefes de família com até & & & & & \\
\hline & sete anos de estudo & & & & & \\
\hline & $<22,2 \%$ (1ㅇ tercil) (Referência) & 812 & 25,5 & 1,00 & & $<0,001$ \\
\hline & Entre $22,2 \%$ e $41,7 \%$ (2o tercil) & 807 & 36,2 & 1,66 * & $1,34-2,05$ & \\
\hline & > 41,7\% (3o tercil) & 803 & 46,3 & 2,52 * & $2,04-3,11$ & \\
\hline & Missing data ** & 4 & & & & \\
\hline & Chefes de familia que recebem até & & & & & \\
\hline & três salários mínimos & & & & & \\
\hline & $<24,5 \%$ (1ㅇ tercil) (Referência) & 807 & 25,8 & 1,00 & & $<0,001$ \\
\hline & Entre $24,5 \%$ e $47,5 \%$ (2o tercil) & 805 & 36,1 & 1,63 * & $1,32-2,02$ & \\
\hline & $>47,5 \%$ (3o tercil) & 810 & 45,9 & 2,45 * & $1,98-3,02$ & \\
\hline & Missing data ** & 4 & & & & \\
\hline Variáveis no & Índice de Theil & & & & & \\
\hline \multirow[t]{14}{*}{ nível do bairro } & $<0,387$ (1ㅇtercil) & 770 & 41,0 & 1,81 * & $1,47-2,23$ & $<0,001$ \\
\hline & Entre 0,387 e 0,482 (2o tercil) & 839 & 39,3 & 1,69 * & $1,37-2,07$ & \\
\hline & > 0,482 (3o tercil) (Referência) & 814 & 27,8 & 1,00 & & \\
\hline & Missing data ** & 3 & & & & \\
\hline & Indicador de condições de vida & & & & & \\
\hline & $<0,873$ (1ㅇtercil) & 795 & 42,5 & 1,87 * & $1,51-2,33$ & $<0,001$ \\
\hline & Entre 0,873 e 0,884 (2o tercil) & 943 & 36,0 & 1,42 * & $1,15-1,77$ & \\
\hline & > 0,884 (3o tercil) (Referência) & 685 & 28,3 & 1,00 & & \\
\hline & Missing data ** & 3 & & & & \\
\hline & Renda per capita em salários mínimos & & & & & \\
\hline & $<1,69$ (1ㅇ tercil) & 805 & 47,6 & 2,72 * & $2,20-3,35$ & $<0,001$ \\
\hline & Entre 1,69 e 3,30 (2o tercil) & 803 & 35,5 & 1,65 * & $1,33-2,04$ & \\
\hline & > 3,30 (3o tercil) (Referência) & 815 & 25,0 & 1,00 & & \\
\hline & Missing data ** & 3 & & & & \\
\hline
\end{tabular}

Fontes: Faerstein et al. 14; Instituto Brasileiro de Geografia e Estatística 15.

* Significativo ( $p$ valor $<0,05$ );

** Missing data: funcionários que residem em bairros e/ou setores sem informação.

veis contextuais no nível de setor censitário, as únicas que apresentaram associação estatisticamente significativa com "SBP ruim" foram o 1o tercil da distribuição da proporção de chefes de família que tinham até sete anos de estudo e o 3o tercil da distribuição da proporção de chefes de família que recebiam até três salários mínimos (Tabela 2). Entre as variáveis contextuais no nível do bairro foi encontrada associação estatisticamente significativa com o 1o e o 2o tercis da distribuição do índice de Theil, o 3o tercil da distribuição do ICV e o 1o tercil da distribuição de renda per capita (Tabela 2).
A “SBP ruim” apresentou tendência linear em relação a todas as variáveis, onde tal estatística se aplica (Tabelas 1 e 2).

Por meio dos CPVs estimados a partir de modelos logísticos hierárquicos vazios com dois níveis de hierarquia, observou-se que a variabilidade da SBP entre os setores censitários foi de $5,07 \%$, enquanto entre os bairros foi de $3,2 \%$, e de $4,23 \%$ entre as regiões administrativas.

Foram ajustados seis modelos não-hierárquicos e seis modelos de regressão hierárquicos com efeitos aleatórios no intercepto. Além de seus coeficientes de regressão, foi estimada tam- 
bém a variabilidade do erro entre os níveis de hierarquia, e para mensurar a parcimônia entre cada modelo foram estimados os respectivos AIC (Akaike Information Criterion).

O modelo mais parcimonioso contendo variáveis contextuais foi o modelo 12 (AIC $=2.596,47$ ). Quando foi ajustado o modelo hierárquico somente com as variáveis sócio-econômicas individuais, a variabilidade entre bairros desapareceu. O mesmo não ocorreu no modelo que incluiu somente as variáveis referentes aos hábitos individuais (Tabela 3 , modelos 8 e 9).

Após o ajuste do modelo hierárquico e nãohierárquico cheio, não foi observada discrepância significativa entre as estimativas dos ORs (Tabela 4).

\section{Discussão}

A utilização da estatística CPV em estudos com desfechos categóricos é útil, por desempenhar papel importante na comparação da quantidade de variação de um determinado desfecho em diferentes níveis contextuais. Neste estudo, apesar de o valor estimado do CPV entre os setores censitários para o estudo da SBP ter sido relevante para a utilização da modelagem hierárquica, os valores das estimativas dos ORs e seus respecti- vos erros padrões foram bastante similares nos modelos de regressão, hierárquicos ou não-hierárquicos. Tal fato pode ter ocorrido devido ao número de unidades dentro do nível mais alto ter sido bastante baixo (em média existia 1,5 indivíduo residindo em cada setor censitário). Devese ressaltar que para tais modelos não é possível mensurar a variabilidade nos níveis contextuais. É necessária uma maior quantidade de observações dentro dos grupamentos, de maneira que seja possível mensurar a homogeneidade dentro de cada grupamento 22 .

Neste estudo, verificou-se que ao ajustar modelos hierárquicos por blocos de variáveis no nível individual, o bloco de variáveis sócio-econômicas tornou nula a variabilidade da SBP decorrente das variáveis no nível do bairro. Uma possível explicação para esse achado é que variáveis sócioeconômicas no nível individual podem ser determinantes para a escolha do local de residência de cada indivíduo. Assim, indivíduos com uma renda alta tendem a escolher um contexto de residência em que a renda média seja alta também. Dessa forma, no nível individual, essa variável já explicaria a variabilidade do desfecho advinda de variáveis explicativas no nível contextual.

Um dos maiores problemas da modelagem de estruturas hierárquicas de dados é a dependência em seus respectivos níveis mais altos de

Descrição dos modelos de regressão de "SBP ruim".

\begin{tabular}{|c|c|c|c|c|c|c|}
\hline \multirow[t]{2}{*}{ Modelos } & & & & \multicolumn{2}{|c|}{ Variância (erro padrão) } & \multirow[t]{2}{*}{ AIC } \\
\hline & & & & Setor & Bairro & \\
\hline Modelos & 1o nível & Modelo 1 & Variáveis demográficas no nível individual & & & $3.121,98$ \\
\hline \multirow[t]{7}{*}{ não-hierárquicos } & & Modelo 2 & Variáveis sócio-econômicas no nível individual & & & $2.930,98$ \\
\hline & & Modelo 3 & Variáveis referentes aos hábitos no nível individual & & & $2.786,48$ \\
\hline & & Modelo 4 & Com todas as variáveis no nível individual & & & $2.630,94$ \\
\hline & & Modelo 5 & Com todas as variáveis no nível individual & & & $2.624,10$ \\
\hline & & & + as no nível do setor censitário & & & \\
\hline & & Modelo 6 & Com todas as variáveis no nível individual & & & $3.121,98$ \\
\hline & & & + as no nível do setor censitário + as no nível do bairro & & & \\
\hline Modelos & 3o nível & Modelo 7 & Variáveis demográficas no nível individual & $0,13(0,11)$ & $0,15(0,05)$ & $3.208,13$ \\
\hline \multirow[t]{7}{*}{ hierárquicos } & & Modelo 8 & Variáveis sócio-econômicas no nível individual & $0,07(0,10)$ & $0,00(0,00)$ & $3.211,22$ \\
\hline & & Modelo 9 & Variáveis referentes aos hábitos no nível individual & $0,06(0,10)$ & $0,09(0,06)$ & $2.768,51$ \\
\hline & & Modelo 10 & Com todas as variáveis no nível individual & $0,07(0,11)$ & $0,00(0,00)$ & $2.625,82$ \\
\hline & 2oo nível & Modelo 11 & Com todas as variáveis no nível individual & $0,08(0,11)$ & & $2.606,17$ \\
\hline & & & + as no nível do setor censitário & & & \\
\hline & & Modelo 12 & Com todas as variáveis no nível individual & $0,10(0,11)$ & & $2.606,17$ \\
\hline & & & + as no nível do setor censitário + as no nível do bairro & & & \\
\hline
\end{tabular}

$\mathrm{AIC}=$ Akaike Information Criterion. 
Modelo logístico hierárquico e não-hierárquico de regressão, com os odds ratio (OR) de "SBP ruim" ajustados por variáveis no nível dos indivíduos e do contexto de residência dos mesmos.

\begin{tabular}{|c|c|c|c|c|c|}
\hline \multirow[t]{2}{*}{ Grupos } & \multirow[t]{2}{*}{ Modelos/Parâmetro } & \multicolumn{2}{|c|}{ Modelo 6} & \multicolumn{2}{|c|}{ Modelo 12} \\
\hline & & OR & IC95\% & OR & IC95\% \\
\hline \multirow[t]{9}{*}{ Demográficas } & Sexo & & & & \\
\hline & Homem & 0,90 & $0,74-1,09$ & 0,90 & $0,73-1,10$ \\
\hline & Mulher (Referência) & 1,00 & & 1,00 & \\
\hline & Idade (anos) & & & & \\
\hline & Até 29 (Referência) & 1,00 & & 1,00 & \\
\hline & $30-39$ & 1,65 * & $1,19-2,29$ & 1,66 * & $1,19-2,32$ \\
\hline & $40-49$ & 1,82 * & $1,29-2,57$ & 1,84 * & $1,30-2,60$ \\
\hline & $50-59$ & 1,96 * & $1,27-3,02$ & 1,98 * & $1,27-3,07$ \\
\hline & Mais de 60 & 2,78 * & $1,28-6,02$ & 2,83 * & $1,29-6,21$ \\
\hline \multirow[t]{14}{*}{ Sócio-econômicas } & Renda & & & & \\
\hline & Até $\mathrm{R} \$ 500,00$ & 2,60 * & $1,42-4,76$ & 2,63 * & $1,42-4,86$ \\
\hline & $\mathrm{R} \$ 501,00-\mathrm{R} \$ 1.000,00$ & 1,48 & $0,93-2,35$ & 1,50 & $0,94-2,39$ \\
\hline & $\mathrm{R} \$ 1.001,00-\mathrm{R} \$ 1.500,00$ & 1,54 * & $1,03-2,30$ & 1,54 * & $1,03-2,33$ \\
\hline & $\mathrm{R} \$ 1.501,00-\mathrm{R} \$ 2.000,00$ & 1,32 & $0,88-1,97$ & 1,32 & $0,88-1,99$ \\
\hline & $\mathrm{R} \$ 2.001,00-\mathrm{R} \$ 3.000,00$ & 1,43 & $0,99-2,08$ & 1,44 & $0,98-2,11$ \\
\hline & $R \$ 3.001,00-R \$ 4.000,00$ & 1,16 & $0,73-1,84$ & 1,16 & $0,73-1,86$ \\
\hline & Mais de R\$ 4.000,00 (Referência) & 1,00 & & 1,00 & \\
\hline & Escolaridade & & & & \\
\hline & 1o grau incompleto & $1,61 *$ & $0,91-2,84$ & 1,63 & $0,92-2,90$ \\
\hline & 1ㅇ grau completo +2 o grau incompleto & 2,25 * & $1,46-3,47$ & 2,29 * & $1,48-3,55$ \\
\hline & 2o grau completo + 3o grau incompleto & 1,62 * & $1,14-2,31$ & 1,64 * & $1,15-2,34$ \\
\hline & 3o grau completo & 1,07 & $0,76-1,51$ & 1,07 & $0,75-1,53$ \\
\hline & Pós-graduação (Referência) & 1,00 & & 1,00 & - \\
\hline \multirow[t]{9}{*}{ Hábitos } & Visita ao dentista & & & & \\
\hline & Nunca vai ao dentista & 0,74 & $0,21-2,60$ & 0,72 & $0,20-2,59$ \\
\hline & Só quando tem problemas & 3,29 * & $2,63-4,11$ & 3,37 * & $2,69-4,23$ \\
\hline & Menos freqüentemente que 2 em 2 anos & 2,73 * & $1,95-3,82$ & 2,79 * & $1,98-3,92$ \\
\hline & De 2 em 2 anos & 1,62 * & $1,20-2,19$ & 1,63 * & $1,20-2,21$ \\
\hline & Pelo menos 1 vez ao ano (Referência) & 1,00 & & 1,00 & \\
\hline & Tabagismo & & & & \\
\hline & Sim & 1,47 * & $1,21-1,80$ & 1,48 * & $1,21-1,81$ \\
\hline & Não (Referência) & 1,00 & & 1,00 & \\
\hline Variáveis no & Domicílios com abastecimento de água & & & & \\
\hline \multirow[t]{10}{*}{ nível do setor } & $<99 \%$ & 1,02 & $0,84-1,24$ & 1,02 & $0,84-1,24$ \\
\hline & $\geq 99 \%$ & 1,00 & & 1,00 & \\
\hline & Chefes de família com até sete anos de es & & & & \\
\hline & $<22,2 \%$ (1ㅇ tercil) (Referência) & 1,00 & & 1,00 & \\
\hline & Entre $22,2 \%$ e $41,7 \%$ (2o tercil) & 1,23 & $0,83-1,84$ & 1,22 & $0,81-1,84$ \\
\hline & $>41,7 \%$ (3o tercil) & 1,24 & $0,75-2,04$ & 1,23 & $0,74-2,05$ \\
\hline & Chefes de familia que recebem até três sa & & & & \\
\hline & $<24,5 \%$ (1ㅇ tercil) (Referência) & 1,00 & & 1,00 & \\
\hline & Entre $24,5 \%$ e $47,5 \%$ (2o tercil) & 0,93 & $0,62-1,39$ & 0,93 & $0,62-1,41$ \\
\hline & $>47,5 \%$ (3o tercil) & 0,94 & $0,56-1,55$ & 0,94 & $0,56-1,58$ \\
\hline
\end{tabular}

(continua) 


\begin{tabular}{|c|c|c|c|c|c|}
\hline \multirow[t]{2}{*}{ Grupos } & \multirow[t]{2}{*}{ Modelos/Parâmetro } & \multicolumn{2}{|c|}{ Modelo 6} & \multicolumn{2}{|c|}{ Modelo 12} \\
\hline & & OR & IC95\% & OR & IC95\% \\
\hline Variáveis no & Índice de Theil & & & & \\
\hline \multirow[t]{11}{*}{ nível do bairro } & $<0,387$ (1ㅇtercil) & 1,12 & $0,78-1,61$ & 1,13 & $0,78-1,64$ \\
\hline & Entre 0,387 e 0,482 (2o tercil) & 1,10 & $0,80-1,50$ & 1,11 & $0,80-1,53$ \\
\hline & > 0,482 (3o tercil) (Referência) & 1,00 & & 1,00 & \\
\hline & Indicador de condições de vida & & & & \\
\hline & $<0,873$ (1으 tercil) & 1,18 & $0,81-1,71$ & 1,18 & $0,81-1,73$ \\
\hline & Entre 0,873 e 0,884 (2o tercil) & 1,22 & $0,91-1,64$ & 1,23 & $0,91-1,64$ \\
\hline & $>0,884$ (3o tercil) (Referência) & 1,00 & & 1,00 & \\
\hline & Renda per capita em salários m & & & & \\
\hline & $<$ 1,69 (1ㅇ tercil) & 1,36 & $0,84-2,20$ & 1,37 & $0,84-2,24$ \\
\hline & Entre 1,69 e 3,30 (2o tercil) & 1,21 & $0,84-1,75$ & 1,22 & $0,84-1,78$ \\
\hline & > 3,30 (3o tercil) (Referência) & 1,00 & & 1,00 & \\
\hline
\end{tabular}

Fontes: Faerstein et al. 14; Instituto Brasileiro de Geografia e Estatística 15.

* Significativo ( $p$ valor $<0,05$ ).

hierarquia. Na maioria das vezes, uma grande quantidade de grupos é mais importante que a quantidade de unidades por grupo, pois, uma quantidade pequena de grupos pode conduzir a estimativas viesadas dos coeficientes dos modelos de regressão hierárquica 23 . No entanto, ignorar a existência de estruturas hierárquicas pode conduzir à subestimação dos erros padrões dos coeficientes de regressão.

Tanto nas análises bivariadas quanto nas multivariadas, as variáveis individuais apresentaram associações mais fortes com a SBP do que as contextuais. É possível que não tenha havido contraste suficiente entre as áreas de residência dos participantes deste estudo, o que limita a generalização desses resultados para outras populações. Vale ressaltar nesse sentido, o percentual de domicílios com abastecimento de água. Essa variável, teoricamente, teria influência na variação da saúde bucal por duas razões: representa um aspecto positivo geral das condições de habitação e acesso ao flúor, já que a água de abastecimento no Rio de Janeiro é fluoretada e o flúor protege a saúde bucal. No entanto, a influência dessa variável contextual pode não ter sido detectada, devido ao acesso quase universal da população estudada à água encanada.

A interpretação das estimativas das variáveis no nível contextual deve ser feita com cautela. Por exemplo, nas associações com as variáveis contextuais índice de Theil e ICV, observou-se leve gradiente em relação à SBP, apesar de não terem sido estatisticamente significativas. Neste estudo observou-se que quanto mais desigual foi a distribuição de renda no local de residência, melhor foi a saúde bucal dos indivíduos que ali residiam. Esse fato pode ser decorrente da desigualdade, nessa população, estar correlacionada positivamente com a renda per capita, i.e., quanto maior foi a desigualdade de renda numa área, também maior foi a renda per capita na mesma (embora essa relação não seja linear). Embora o mesmo resultado não tenha sido encontrado na associação entre a desigualdade e as condições de vida (ICV), observamos que a correlação entre o ICV e a renda per capita foi positiva. Isso poderia ser explicado pelo fato da população de estudo residir em contextos de maior renda per capita, o que facilitaria a aquisição de serviços e produtos de melhor qualidade, incluindo-se os serviços de saúde e maior acesso à informação, entre outros fatores que dependem dessa característica. Dessa maneira, indivíduos com renda baixa acabariam se beneficiando por residirem em áreas onde residem indivíduos com renda alta e condições de vida melhores, usufruindo serviços e produtos similares aos utilizados por aqueles indivíduos com renda mais alta. 


\section{Resumo}

Um problema dos delineamentos ecológicos é o viés de agregação. Uma alternativa é selecionar estruturas contextuais de menor tamanho e maior homogeneidade interna. Nós comparamos diferentes estruturas geográficas de dados, com a finalidade de estimar efeitos de variáveis sócio-econômicas contextuais na chance de saúde bucal percebida ruim. As análises foram baseadas em um delineamento misto, que compreendeu os participantes dos censos Pró-Saúde I e II, residentes no Município do Rio de Janeiro, Brasil, em 1999 ( $n=2.426$ ). Os indicadores do contexto de residência dos participantes foram extraídos do Censo Demográfico da Unidade Federativa do Rio de Janeiro de 1991. Em estudos epidemiológicos com estruturas hierárquicas de dados e desfechos categóricos, a utilização do coeficiente de partição de variância permite analisar a origem da variabilidade do desfecho em relação aos níveis hierárquicos. Neste estudo, a partição geográfica de menor tamanho (setor censitário) foi a melhor unidade contextual de análise para explicar a variabilidade da saúde bucal percebida. Atributos sócio-econômicos individuais revelaram maior poder explicativo para a variação na saúde bucal percebida do que os contextos sócio-econômicos das áreas de residência.

Saúde Bucal; Análise Sócio-econômica; Adulto

\section{Colaboradores}

W. S. Tassinari e A. P. de León revisaram a literatura referente à análise multinível na área da saúde, participaram da alocação de cada participante à sua área de residência e da construção indicadores sócio-econômicos contextuais de acordo com a demarcação do censo demográfico, participaram também da análise estatística dos dados, da interpretação dos resultados e da redação do artigo. G. L. Werneck orientou a coleta dos dados individuais do estudo Pró-Saúde, orientou a análise estatística dos dados, interpretou os resultados, escreveu o artigo. E. Faerstein, C. S. Lopes e D. Chor participaram da coleta dos dados individuais do Estudo Pró-Saúde e da redação do artigo. P. Nadanovsky revisou a literatura referente à associação entre fatores sócio-econômicos e saúde bucal, à saúde bucal percebida e à análise multinível na saúde bucal, interpretou os resultados da análise estatística dos dados, escreveu o artigo.

\section{Referências}

1. Susser M. The logic in ecological: I. The logic of analysis. Am J Public Health 1994; 84:825-9.

2. Greenland S, Robins J. Invited commentary: ecologic studies - biases, misconceptions, and counterexamples. Am J Epidemiol 1994; 139:747-61.

3. Morgenstern H. Ecologic studies. In: Rothman KJ, Greenland S, editors. Modern epidemiology. 2nd Ed. Philadelphia: Lippincott-Raven; 1998. p. 459-80.

4. Goldstein H. Multilevel statistical models. London: Edward Arnold; 1995.

5. Leyland AH, McLeod A. Mortality in England and Wales, 1979-1992. An introduction to Multilevel Modelling using MLwiN. Glasgow: MRC Social and Public Health Sciences Unit; 2000. (Occasional Paper, 1).

6. Jelinski DE, Goodchild M, Steyaert L. The modifiable areal unit problem and implications for landscape ecology. Landscape Ecology 1996; 11: 129-40.

7. Locker D. Measuring social inequality in dental health services research: individual, household and area-based measures. Community Dent Health 1993; 10:139-50.
8. Locker D. Deprivation and oral health: a review. Community Dent Oral Epidemiol 2000; 28:161-9.

9. Nadanovsky P, Sheiham A. The relative contribution of dental services to the changes and geographical variations in caries status of 5- and 12-year-old children in England and Wales in the 1980s. Community Dent Health 1994; 11:215-23.

10. Nadanovsky P, Sheiham A. Relative contribution of dental services to the changes in caries levels of 12-year-old children in 18 industrialized countries in the 1970s and early 1980s. Community Dent Oral Epidemiol 1995; 23:331-9.

11. Gilthorpe MS, Maddick IH, Petrie A. Introduction to multilevel modelling in dental research. Community Dent Health 2000; 17:222-6.

12. Pattussi MP, Marcenes W, Croucher R, Sheiham A. Social deprivation, income inequality, social cohesion and dental caries in Brazilian school children. Soc Sci Med 2001; 53:915-25.

13. Baldani MH, Vasconcelos AGG, Antunes JLF. Associação do índice CPO-D com indicadores sócioeconômicos e de provisão de serviços odontológicos no Estado do Paraná, Brasil. Cad Saúde Pública $2004 ; 20: 143-52$. 
14. Faerstein E, Lopes CS, Valente K, Sole PM, Ferreira MB. Pré-testes de um questionário multidimensional autopreenchível: a experiência do estudo Pró-Saúde. Physis (Rio J) 1999; 7:117-30.

15. Instituto Brasileiro de Geografia e Estatística. Censo demográfico do Município do Rio de Janeiro. Rio de Janeiro: Instituto Brasileiro de Geografia e Estatística; 1991.

16. Browne WJ, Subramanian SV, Jones K, Goldstein H. Variance partitioning in multilevel logistic models that exhibit over-dispersion. J R Stat Soc Ser A Stat Soc 2005; 168:599-614.

17. Afonso-Souza G, Nadanovsky P, Chor D, Faerstein E, Werneck GL, Lopes CS. Self-perceived oral health and routine dental visits in Brazilian adults. J Dent Res 2005; 84 (Spec Iss A):731.

18. Neri M, Camargo JM. Structural reforms, macroeconomic fluctuations and income distribution in Brazil. Santiago: Economic Commission for Latin America and the Caribbean, United Nations; 1999. (Série Reformas Económicas, 39).
19. Motta GO. Pobreza, desigualdade e heterogeneidade nas favelas do Rio de Janeiro [Monografia de Graduação]. Rio de Janeiro: Instituto de Economia, Universidade Federal do Rio de Janeiro; 2000.

20. Programa das Nações Unidas para o Desenvolvimento. Desenvolvimento humano e condições de vida: indicadores brasileiros. Brasília: Programa das Nações Unidas para o Desenvolvimento; 1998.

21. Rodriguez G, Goldman N. An assessment of estimation procedures for multilevel models with binary responses. J R Stat Soc Ser A Stat Soc 1995; 158:73-90.

22. Carvalho MS, Cruz OG, Nobre FF. The use of multivariate cluster analysis and aggregation algorithm to propose a new spatial partitioning of Rio de Janeiro, Brazil. Stat Med 1996; 15:1885-94.

23. Maas CJM, Hox JJ. Sample sizes for multilevel modeling. Am J Public Health 1999; 89:1181-6.

Recebido em 18/Ago/2005

Versão final reapresentada em 16/Fev/2006 Aprovado em 02/Mai/2006 